\title{
How Yeast Can Inform Us about Healthy Aging
}

\author{
Ian Macreadie, Yen Nhi (Rachel) Luu \\ School of Science, RMIT University, Melbourne, Australia \\ Email: ian.macreadie@rmit.edu.au
}

How to cite this paper: Macreadie, I. and Luu, Y.N. (2018) How Yeast Can Inform Us about Healthy Aging. Open Journal of Social Sciences, 6, 24-31. https://doi.org/10.4236/jss.2018.66003

Received: January 31, 2018

Accepted: May 20, 2018

Published: May 23, 2018

\begin{abstract}
Yeasts are eukaryotes like us, and they have informed us about our cellular and molecular biology for many decades. They are unicellular and live with 6000 genes, carrying out many of the same processes that we do. Like us, yeast exhibit the same processes of aging, with telomere shortening, loss of mitochondrial function, reduced proteostasis, reduced robustness and stress. Some of these attributes are associated with aging and may not be the cause of aging. Therefore, it is important to consider attributes that clearly affect the fitness of cells. We have constructed yeast with a reporter of deleterious protein turnover. It involves the Alzheimer's amyloid beta peptide fused to a green fluorescent protein to aid its visualization in living cells. The use of this reporter enables high throughput assays to find compounds that can improve proteostasis in older cells. Compounds, like simvastatin, improve proteostasis and improve health outcomes inageing. Stress and biochemicals may decrease health and lifespan. Yeast can be used to study aging, drugs and stress, and to search for compounds that improve robustness in cells affected by drugs or stress.
\end{abstract}

\section{Keywords}

Genome Maintenance, Mitochondrial Function, Protein Folding, Protein Turnover, Saccharomyces cerevisiae, Stress

\section{Introduction}

The study of cellular and molecular biology is not at all easy in humans. Our knowledge tends to come from:

- observations of people with natural "mutations";

- observations of people with respect to lifestyles and diets;

- from experiments involving mammalian cell culture;

- from experiments with mice;

- from extrapolation from yeast and other model organisms, like the worm 


\section{Caenorhabditis elegans.}

Extrapolation from yeast may come as a surprise to the reader. In fact, yeast has contributed more to the knowledge of our basic biology than any other organism. Some recognition of this is seen in the large proportion of Nobel Prizes in Medicine and Chemistry that have been awarded for studies in yeast [1] [2], most often budding yeast, which represents the majority of yeast.

While a yeast culture may seem immortal, the population within it is very heterogeneous, and a small fraction of the cells will be dead. Most yeast species exhibit vegetative growth by budding. Cells grow and reach a point where they divide by giving rise to new growth in a bud which eventually releases from the mother to form an independent new cell. Throughout their life cells continue to bud and with each bud they produce, there is a bud scar, so the age of the yeast (that is, how many buds she has produced) can be measured by the number of bud scars on the surface (see Figure 1). Using modern technologies, it is possible to study sub-populations of different age within a yeast culture.

\section{Ease of Use}

\subsection{Yeast Culture and Single Cell Studies}

Yeasts have been cultured successfully for millennia in the manufacture of beer, wine and bread. Their culture is inexpensive and can utilize rich media as well as

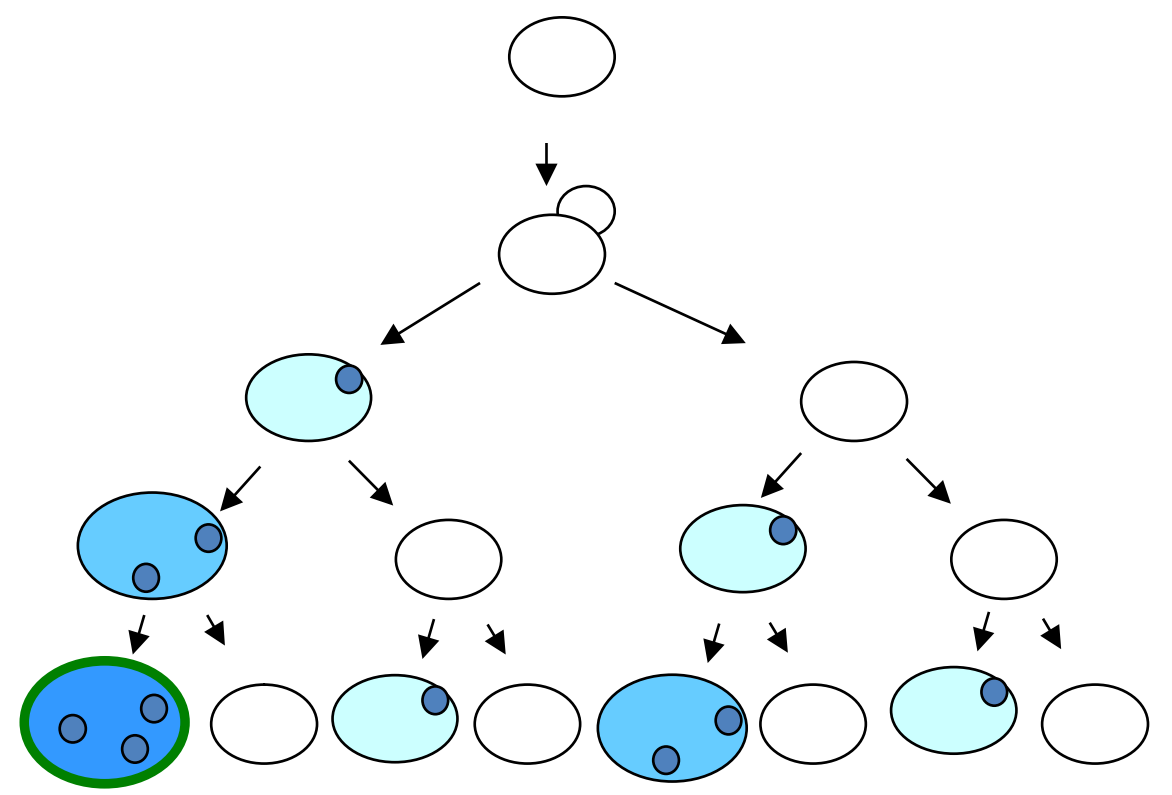

Figure 1. Growth of the budding yeast Saccharomyces cerevisiae seen in three generations. The first cell grows a bud which breaks off to leave a mother and daughter cell. The mother is left with a bud scar. Both repeat the process and each new mother is left with a bud scar. A third division is also shown, resulting in eight cells (bottom line) comprising four young cells without bud scars. The mothers of these cells may be relatively just one generation older (with one bud scar), and older mother cells with two or more bud scars. The oldest cell, with three bud scars, displays signs of aging (discussed in the text). Note that this diagram predicts a older cells to comprise $\sim 12 \%$ of the population. 
chemically defined synthetic media. This can ensure safety as well as the opportunity to extensively modify their nutritional environment to investigate effects on yeast health. In comparison, the culture of human cells is expensive and usually requires serum and/or growth factors, and these components need to be verified as being free of viruses and prions that could harm the researcher. Cell lines are often immortalized and not representative of primary cells that have a limited lifespan. Some of the most important cells for ageing studies, neuronal cells, are terminally differentiated and not normally amenable to cell culture.

Looking at a yeast culture is somewhat like looking at our own population, in that there is a diversity of ages. In interrogating the aging human population, we analyse older people versus younger people: we can do the same comparison with yeast. This can be done with analytical flow cytometry, where the population is analyzed one cell at a time by high-speed, high throughput machines that can measure and record the size, color, density, etc. of 10,000 cells or more in minutes. This information can then be further analyzed. For example, we might ask how the oldest cells are affected by treatment with a particular compound.

Preparative flow cytometers (cell sorters) can help separate populations of cells so that they can be subjected to further examination, such as genome wide expression analyses.

\subsection{Yeast Genetics}

Yeast genetics have been studied for almost a century. Auxotrophic mutations in yeast were obtained to decipher biochemical pathways and they were freely distributed between yeast genetics laboratories throughout the world, rapidly aiding our knowledge of biochemical pathways. Yeast genetics greatly aided the emerging area of molecular biology in the 1980s and helped the establishment of gene and gene product relationships. It was convenient that yeast had very few introns, so the elucidation of the structure of the structure of the yeast nuclear genome in 1996, the first eukaryotic genome to be fully sequenced, was more straightforward.

Since that time reverse genetics has been at play and the effects of most genes are now known. Such information has been used to inform about human genes with similar encoded functions. With the availability of yeast deletion libraries, we have a systematic view that many genes affect the lifespan of yeast, and many affect the health of cells.

\subsection{Yeast Mitochondrial Genome}

The mitochondrion is known as the powerhouse of the cell, and in humans its function declines with age, leading to cell death. In contrast, mitochondrial function in yeast is not obligatory but is dispensable. Loss of mitochondrial function in yeast simply means a loss of respiration, so the cells cannot grow on glycerol or ethanol. However, their growth is still reasonable on glucose. This yeast attribute improves the amount of information one can derive from yeast. 
Many gene functions are required for stability of the mitochondrial genome, and it is clear that many mutations lead to high instability of the mitochondrial genome. Likewise, exposure to certain drugs or environments can lead to mitochondrial genome instability, including total loss of the mitochondrial genome.

\subsection{Telomere Maintenance}

Our cells are composed of linear chromosomes that need to be faithfully copied and transmitted to the new cells formed by cell division. Most of the DNA is copied by a robust replication process where the copies are like mirror images of the originals, a so-called DNA template dependent process. However, the copying of the ends of the chromosomes, telomeres, is a special challenge, since it cannot use this process. Instead an enzyme, telomerase, orchestrates the building of the ends which contain highly repeated sequence of TTAGGG repeated as many as 3000 times. With age, it is noticed that telomeres shorten, so that by 40 years of age telomeres can be half their original length. Interestingly, immortalized cells like many cancers keep longer telomeres and overproduce telomerase. For this reason, there is interest in regulating telomerase as a possible intervention in aging. Also, there is interest in monitoring telomerase as cancer biomarker, and targeting it in treatment of cancer. There is also interest in overproducing telomerase to help preserve mammalian cell lines that are used in research throughout the world.

So far regulation of telomerase has not led to changes in longevity of animals or people.

\subsection{Proteostasis}

For the maintenance of cells there is a need to keep cellular components in equilibrium. This can be achieved through the processes of proteostasis (protein degradation through autophagy, proteosomal degradation, unfolded protein response, etc.). Cells degrade proteins efficiently throughout our life, however, as we age proteostasis starts to fail. It is notable that in many diseases associated with ageing there is buildup of some proteins and this may account for the loss of many cell types, particularly neuronal cells, in the elderly. For example, in Alzheimer's disease there is an accumulation of amyloid beta (A $\beta$ ), in Parkinson's disease an accumulation of $\alpha$-synuclein, and in Huntington's disease an accumulation of huntingtin in Huntington's disease. A $\beta, \alpha$-synuclein and huntingtin are proteins that are deleterious and their build-up leads to stress on cells.

These processes can also be observed in yeast by tagging these problematic proteins with a fluorescent tag to enable their visualization in cells. Yeast cells were engineered to produce $\mathrm{A} \beta$ tagged with green fluorescent protein (to make GFP-A $\beta$ ), enabling it to be produced and seen in whole yeast cells [3]. It can be seen in Figure 2 that in young yeast cells the GFP-A $\beta$ is degraded while in the older cells it persists. This shows old yeast to experience the same problems with proteostasis as old people. 


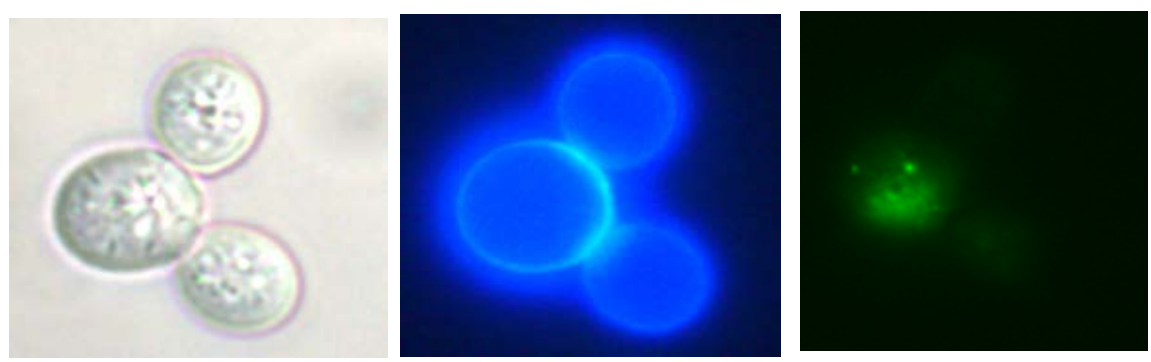

Figure 2. Ageing and protein turnover in yeast. Left panel shows a mother cell (the largest cell), with two buds, still attached. The middle panel shows calcofluor staining of two bud scars on the mother. The right panel shows GFP-A $\beta$ in the mother: the daughter cells have received cytoplasm from the mother but have removed the GFP-A $\beta$.

About $12 \%$ of the population ("grandparent" yeast and older with 2 or more bud scars) exhibit aging. Without any need for ethics approval, this population can be readily subjected to all sorts of treatments to find conditions that improve proteostasis (or that make it worse).

Recently we have found that the cholesterol lowering drug, simvastatin, improves protein turnover in old yeast cells (M. Subhan, K. Gardiner, R. Faryal and I. Macreadie, unpublished). In an untreated population, GFP-Aßwas visualized in $12 \%$ of yeast cells, but growth in the presence of simvastatin reduced this level to $0.6 \%$ of the population. In other words, simvastatin aided clearance of the deleterious GFP-A $\beta$ in $95 \%$ of the older population. In parallel to this work two other statins, atorvastatin and lovastatin, were also tested. Atorvastatin and lovastatin also significantly aided clearance of GFP-A $\beta$, but the reductions were not as great as those induced by simvastatin: atorvastatin led to a $60 \%$ decrease and lovastatin led to a $20 \%$ decrease in GFP-A $\beta$. These results in yeast are extremely informative about a role for statins in proteostasis in old yeast cells.

Interestingly, in human cell lines and animal studies, statins have been shown to increase autophagy [4] [5] [6] [7] [8] and the unfolded protein response [9] [10].

Most remarkable however, is that in a large epidemiological study of millions of people, simvastatin stands out among statins as a drug that lowers the incidence of Alzheimer's and Parkinson's disease [11], which are leading causes of age-related morbidity. The yeast study, for the first time, suggests that statins might exert their protective effect through removal of $\mathrm{A} \beta$.

Further work is required to determine whether simvastatin might be exerting a protective effect in Parkinson's disease through the removal of a deleterious protein like $\alpha$-synuclein.

\section{Stress}

Stress has a major impact on our health and our survival. Stress may result from age, nutritional deprivation, mutations and environmental conditions, such as extreme cold or heat. Studies on care-givers show that the physical and psychological stresses they encounter decrease their survival [12] [13]. There are vari- 
ous biological blood markers, usually proteins, that can be used as indicators of stress levels. The measurement of such markers is made by pathology labs who compare levels in test samples with a reference range. It is important to keep daily stress at low levels to maintain good health. Continued high stress leads to a shorter lifespan and increased risk of disease.

Yeasts, like other microbes, are masters in adapting to meet challenges and there is much we can learn from them. Their vast numbers in a population, and their variability due to mutation usually means that a variant is present that may survive a new challenge. Although it is not currently practical for humans to use this approach, we can learn from the yeast and possibly improve our resistance through pharmaceutical and nutraceutical interventions.

Yeast also activates genes to help cope with the stress. Using genome wide expression analyses we can find out how they respond to the stress. Many genes are activated by stress and they have become known as stress-response genes. The same set of genes is induced by heat stress, reactive oxygen species and misfolded proteins. This set each have a specific "heat shock element" (HSE) upstream of the genes that respond to these stresses. They are all "switched on" in cells producing GFP-Aß, aiding in defense against the stress of this deleterious protein.

Mounting stress responses may protect against stress but if there is no stress, the stress response may itself be harmful. For example, it is apparent that autoimmune diseases turn defense system against the body. Likewise, chronic inflammation appears to cause major debilitating diseases like chronic fatigue, fibromyalgia and arthritis.

In addition, there are other things to note in the analysis of the genome wide expression. One of the most highly induced genes, $A H P 1$, encodes an allyl hydroperoxidase that is involved in detoxification of toxic lipid peroxides. This suggests oxidation of lipids may be involved in $\mathrm{A} \beta$ toxicity.

The presence of GFP-A $\beta$ induces stress in yeast and this can be measured by genome wide expression, or by reporter assays. For example, linking the HSE to a reporter gene like lac $Z$ allows the detection of the lacZgene product, $\beta$-galactosidase, when cells are stressed. It can be seen that if the formation of $\mathrm{A} \beta$ oligomers is inhibited, the cells are less stressed [3]. Likewise, this system can be used to screen for compounds that decrease stress on cells.

\section{Dealing with Toxic Natural Biochemical and Pharmaceuticals}

Our bodies produce and rely on thousands of biochemicals, including some that are toxic. One such example is the neurotransmitter dopamine. Dopamine is essential and is produced by dopaminergic neurons, however, the loss of these neurons results in the age-related Parkinson's disease (PD). PD is treated with L-DOPA, which is converted to dopamine in the brain, restoring neurotransmission. Mammalian cell studies show that dopamine is actually toxic. Yeast 
studies reveal that dopamine toxicity targets mitochondrial function, conveniently demonstrated in yeast by showing dopamine inhibits respiratory growth. Further, yeast demonstrate that protection from this inhibition can be obtained through the use of antioxidants like glutathione and ascorbate [14].

Other biochemicals that have been examined in yeast studies include biogenic amines, like tyramine (C. Kadkol and I. Macreadie, unpublished) and phenyl ethylamine [15], L-DOPA [16], and drugs like fluoxetine (K. Spencer-Bakes and I. Macreadie, unpublished).

\section{Conclusions}

Yeast can be used for many studies on aging and cell health. Yeast offers convenient handling and quick results.

Study of the cholesterol lowering drug, simvastatin, indicates that it also aids the clearance of a deleterious protein, and probably also does this in people, suggesting this may account for its beneficial effects in $\mathrm{AD}$ and $\mathrm{PD}$.

Stress can be observed in yeast, as well as agents that increase or decrease stress in yeast.

Yeast can be used to study toxic biochemicals, as well as ways to reduce the toxicity of such biochemicals.

Although some extrapolation is called for in applying yeast knowledge to human health, the lessons learned from yeast are valuable.

\section{References}

[1] Macreadie, I.G. (2017) Yeast as a Model Organism for the Pharmaceutical and Nutraceutical Industries. Microbiology Australia, 38, 55-57.

[2] Macreadie, I. (2017) Yeasts, Molecular and Therapeutic Applications. Kirk-Othmer Encyclopedia of Chemical Technology [Internet]. John Wiley \& Sons, Inc., 1-24. https://doi.org/10.1002/0471238961.yeasmacr.a01.pub2

[3] Caine, J., Sankovich, S., Antony, H., Waddington, L., Macreadie, P., Varghese, J. and Macreadie, I. (2007) Alzheimer's A $\beta$ Fused to Green Fluorescent Protein Induces Growth Stress and a Heat Shock Response. FEMS Yeast Research, 7, 1230-1236. https://doi.org/10.1111/j.1567-1364.2007.00285.x

[4] Huang, B., Jin, M., Yan, H., Cheng, Y., Huang, D., Ying, S. and Zhang, L. (2015) Simvastatin Enhances Oxidized Low Density Lipoprotein Induced Macrophage Autophagy and Attenuates Lipid Aggregation. Molecular Medicine Reports, 11, 1093-1098. https://doi.org/10.3892/mmr.2014.2790

[5] Vilimanovich, U., Bosnjak, M., Bogdanovic, A., Markovic, I., Isakovic, A., Kravic-Stevovic, T., Mircic, A., Trajkovic, V. and Bumbasirevic, V. (2015) Statin-Mediated Inhibition of Cholesterol Synthesis Induces Cytoprotective Autophagy in Human Leukemic Cells. European Journal of Pharmacology, 765, 415-428. https://doi.org/10.1016/j.ejphar.2015.09.004

[6] Vilimanovich, U., Bosnjak, M., Bogdanovic, A., Markovic, I., Isakovic, A., Kravic-Stevovic, T., Mircic, A., Trajkovic, V. and Bumbasirevic, V. (2015) Statin-Mediated Inhibition of Cholesterol Synthesis Induces Cytoprotective Autophagy in Human Leukemic Cells. European Journal of Pharmacology, 765, 415-428. https://doi.org/10.1016/j.ejphar.2015.09.004 
[7] Gu, W., Cui, R., Ding, T., Li, X., Peng, J., Xu, W., Han, F. and Guo, X. (2017) Simvastatin Alleviates Airway Inflammation and Remodelling through Up-Regulation of Autophagy in Mouse Models of Asthma. Respirology, 22, 533-541. https://doi.org/10.1111/resp.12926

[8] Gao, K., Wang, G., Wang, Y., Han, D., Bi, J., Yuan, Y., Yao, T., Wan, Z., Li, H. and Mei, X. (2015) Neuroprotective Effect of Simvastatin via Inducing the Autophagy on Spinal Cord Injury in the Rat Model. Biomedical Research International, 2015, 260161. https://doi.org/10.1155/2015/260161

[9] Chen, J.C., Wu, M.L., Huang, K.C. and Lin, W.W. (2008) HMG CoA Reductase Inhibitorsactivate the Unfolded Protein Response and Induce Cytoprotective GRP78 Expression. Cardiovascular Research, 80, 138-150.

https://doi.org/10.1093/cvr/cvn160

[10] Holstein, S.A. and Hohl, J. (2011) Isoprenoid Biosynthetic Pathway Inhibition Disrupts Monoclonal Protein Secretion and Induces the Unfolded Protein Response Pathway in Multiple Myeloma Cells. Leukaemia Research, 35, 551-559. https://doi.org/10.1016/j.leukres.2010.08.008

[11] Wolozin, B., Wang, S.W., Li, N.C., Lee, A., Lee, T.A. and Kazis, L.E. (2007) Simvastatin Is Associated with a Reduced Incidence of Dementia and Parkinson's Disease. BMC Medicine, 5, 20. https://doi.org/10.1186/1741-7015-5-20

[12] Schulz, R. and Beach, S.R. (1999) Caregiving as a Risk Factor for Mortality: The Caregiver Health Effects Study. JAMA, 282, 2215-2219. https://doi.org/10.1001/jama.282.23.2215

[13] Fredman, L., Cauley, J.A., Hochberg, M., Ensrud, K.E. and Doros, G. (2010) Mortality Associated with Caregiving, General Stress, and Caregiving-Related Stress in Elderly Women: Results of Caregiver-Study of Osteoporotic Fractures. Journal of the American Geriatrics Society, 58, 937-943. https://doi.org/10.1111/j.1532-5415.2010.02808.x

[14] Macreadie, I.G., Bartone, N. and Sparrow, L. (2010) Inhibition of Respiratory Growth and Survival in Yeast by Dopamine and Counteraction with Ascorbate or Glutathione. Journal of Biomolecular Screening, 15, 297-301. https://doi.org/10.1177/1087057109358920

[15] Phillips, J. and Macreadie, I. (2017) Inhibition of Respiration in Yeast by 2-phenylethylamine. Current Bioactive Compounds, 13.

[16] Escorial, C. and Macreadie, I. (2018) Comparison of Cytocidal Activities of L-DOPA and Dopaminein S. cerevisiae and C. glabrata. Current Bioactive Compounds, 14, in Press. https://doi.org/10.2174/1573407214666180108144216 